\title{
Topical Prostaglandin Analogues and Conjunctival Inflammation in Uveitic Glaucoma
}

\author{
Simon R.J. Taylor ${ }^{*}, 1,2,3$, Avinash Gurbaxani ${ }^{3}$, Ahmed Sallam $^{3}$ and Sue Lightman ${ }^{2,3,4}$ \\ ${ }^{I}$ Division of Immunology \& Inflammation, Faculty of Medicine, Imperial College London, London, UK \\ ${ }^{2}$ Royal Surrey County Hospital NHS Foundation Trust, Guildford, Surrey, UK \\ ${ }^{3}$ Moorfields Eye Hospital, City Road, ECIV 2PD, London, UK \\ ${ }^{4} U C L$ Institute of Ophthalmology, 11-43 Bath Street, EC1V 9EL, London, UK
}

\begin{abstract}
Purpose: A pilot study to determine whether topical prostaglandin analogues alter the expression of conjunctival inflammatory markers in patients with uveitic glaucoma.

Methods: Prospective, single-masked case series of 20 patients with uveitis and secondary raised intraocular pressure. Participants were divided into four groups of five patients dependent on their use of topical medication: (1) prostaglandin analogues only, (2) corticosteroids only, (3) both prostaglandin analogues and corticosteroids, (4) no topical medication. Conjunctival cells were harvested by impression cytology and were examined for inflammatory markers (CD3, CD54, HLA-DR, CCR4, CCR5) by flow cytometry. A tear fluid sample was also examined for inflammatory cytokines (IL12p70, IL-2, IL-10, IL-8, IL-6, IL-4, IL-5, IFN-gamma, IL-1beta, IFN-alpha, IFN-beta) by multiplex bead arrays.

Results: All groups demonstrated increased markers of conjunctival inflammation. There was no significant difference in levels of any inflammatory markers between the four groups, suggesting that the use of topical prostaglandin analogues does not increase conjunctival levels of inflammation beyond those already seen in uveitis

Conclusions: The use of topical prostaglandins does not appear to induce conjunctival inflammation over that which is already present in patients with uveitic glaucoma. This supports the use of topical prostaglandin analogues in patients with uveitic glaucoma, indicating that their use is unlikely to adversely affect subsequent glaucoma filtration surgery through the induction of chronic conjunctival inflammation.
\end{abstract}

Keywords: Uveitic glaucoma, prostaglandin analogues, conjunctival inflammation, trabeculectomy.

\section{INTRODUCTION}

Secondary glaucoma is seen in about $10-20 \%$ of patients attending a uveitis clinic and occurs in combination with all types of uveitis [1-4]. In spite of aggressive medical therapy, progressive visual field loss and optic nerve damage can occur in up to a third of patients, requiring surgical intervention in the form of trabeculectomy or tube drainage surgery [5]. It is known that chronic use of topical antiglaucoma medication is associated with an increased rate of failure of trabeculectomy in primary open angle glaucoma $[6,7]$, but whether this is significant in uveitic glaucoma is unknown.

The prostaglandin analogues are a group of compounds that have become the most frequently used ocular hypotensive drugs owing to their efficiency in reducing intraocular pressure and the low incidence rate and severity of adverse events, in particular in the absence of systemic side effects, especially in comparison to the beta-blockers [810]. However, they are pro-inflammatory, at least in theory,

*Address correspondence to this author at the Imperial College London Faculty of Medicine, Room 5N8B, 5th Floor Commonwealth Building, Hammersmith Hospital, London W12 0NN, UK; Tel: +44 (0) 208383 2306; Fax: +44 (0) 207566 2266; E-mail: s.r.taylor@imperial.ac.uk and cause conjunctival side-effects including hyperaemia, in $10-60 \%$ of cases $[8,9,11-14]$.

The purpose of this pilot study was to address the effects of prostaglandin analogues on the expression of inflammatory markers on the conjunctival epithelium and tear film in patients who suffer from uveitis, in order to determine whether these drugs are likely to adversely prejudice subsequent trabeculectomy surgery in this group.

\section{METHODS}

This pilot study was a prospective, single-masked, comparative case series of 20 patients with an underlying diagnosis of non-infectious uveitis and secondary raised intraocular pressure. The study was approved by the UCLH Research Ethics Committee (06/Q0512/10). Patients were recruited into four groups on the basis of their use of topical medication (topical prostaglandins only; topical corticosteroids only; both topical prostaglandins and topical corticosteroids; and no drops, which served as a control group). Topical medication used by patients enrolled in this study included the corticosteroids G. Dexamethasone 0.1\% (Maxidex, Alcon, London, UK) and G. Rimexolone 1\% (Vexol, Alcon, London, UK), and the prostaglandin analogues G. Latanoprost $0.003 \%$ (Xalatan, Pfizer, Surrey, UK) and G. Travoprost $50 \mu \mathrm{g} / \mathrm{ml}$ (Travatan, Alcon, London, UK). 
The topical corticosteroid groups were enrolled in order to determine the impact of topical corticosteroids on cytokine expression, both with and without concomitant prostaglandin administration. In order to increase the probability that the results reflected current treatment, patients were excluded if they were on other topical or systemic medication, or if their topical medication had not been stable for at least one month. Five patients were enrolled into each group.

Both conjunctival cells and tear samples were tested in order to improve the likelihood of detecting any differences between the groups. Both the sampling and the laboratory tests were performed by a single investigator masked as to the groups. A tear fluid sample was obtained prior to impression cytology by capillary tube (Drummond Scientific Co., Broomall, PA, US) and stored at $-70^{\circ} \mathrm{C}$. Conjunctival cells were then harvested by impression cytology as described by Baudouin [15] using $0.20 \mu \mathrm{m}$ polyether-sulfone filter papers (Supor 200, Pall Corporation, UK). Two pieces of filter paper measuring $13 \mathrm{~mm} \times 6.5 \mathrm{~mm}$ were applied to the superior bulbar conjunctiva for 15 seconds after application of local anaesthetic drops and then fixed in $0.05 \%$ paraformaldehyde.

Conjunctival cells were obtained by spinning down the cells from the filters. Cells were divided and stained with directly conjugated antibodies to $\mathrm{CD} 3^{\mathrm{FITC}}$, CD54 $4^{\mathrm{PE}}$, HLA$\mathrm{DR}^{\mathrm{PerCP}}, \mathrm{CCR} 4^{\mathrm{PE}}$ and CCR5 ${ }^{\mathrm{FITC}}$ (Becton Dickinson, Cowley, UK). They were then examined by flow cytometry using a Becton Dickinson FACSCalibur machine (BD, San Jose, CA, US). A minimum of 1,000 counts was considered to be acceptable and at least this number was collected per sample. Data was analyzed using Winlist (Verity, USA)

Tear samples were then examined for inflammatory cytokines using the same FACSCalibur flow cytometer. A multiplex kit (Bender Medsystems, Austria) was used according to manufacturer's instructions and the data analyzed using their dedicated software in order to detect expression of IL-12p70, IL-2, IL-10, IL-8, IL-6, IL-4, IL-5, IFN- $\gamma$, IL- 1 beta, IFN- $\alpha$ and IFN- $\beta$. Kruskal Wallis tests were used to compare the surface expression levels and cytokine levels between the four groups.

\section{RESULTS}

We first examined the results of impression cytology of the conjunctiva. All of the inflammatory markers measured (CD3, CD54, HLADR, CCR4 and CCR5) were found to be increased above normal levels in all of the groups, but there was no significant difference in the levels seen between any of the groups (Fig. 1), suggesting that neither topical prostaglandins nor topical corticosteroids have a significant additional effect on conjunctival surface markers of inflammation in patients with uveitis. This supports the conclusion that topical prostaglandins do not significantly increase conjunctival inflammation in these patients.

Similar results were seen from the examination of the tear fluid (Fig. 2). Increased expression of inflammatory markers was seen in all the groups, but there was no significant increase seen in the groups taking topical prostaglandins. This again supports the conclusion that topical prostaglandins do not significantly increase
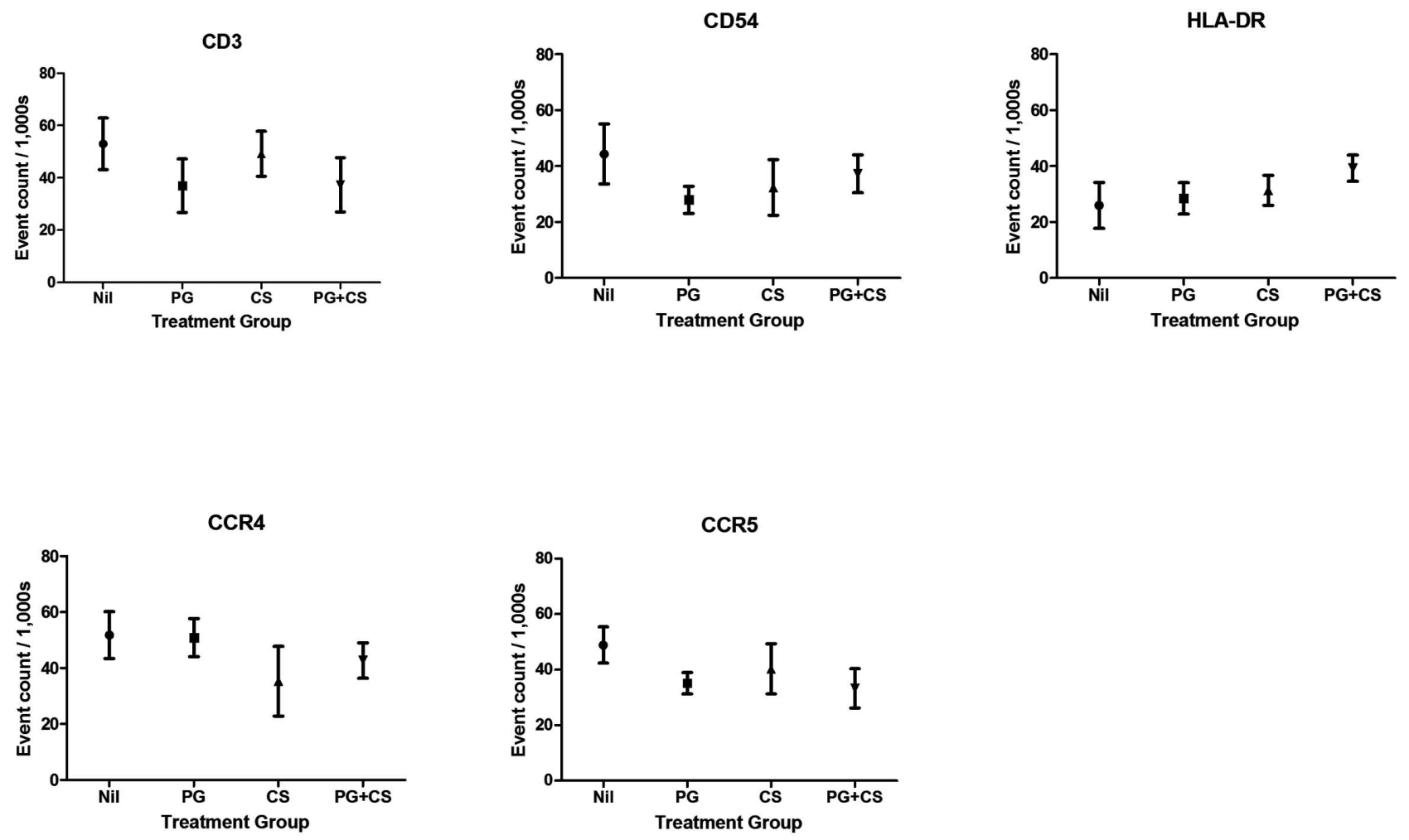

Fig. (1). Conjunctival markers of inflammation. Conjunctival cells were obtained by impression cytology, stained and assessed by flow cytometry. Nil, no topical medication; PG, prostaglandin analogues only; CS, corticosteroids only; PG+CS, prostaglandin analogues and corticosteroids concurrently. The mean \pm the standard error of the mean of the flow cytometric event counts obtained is presented. There were no statistically significant differences between the groups. 

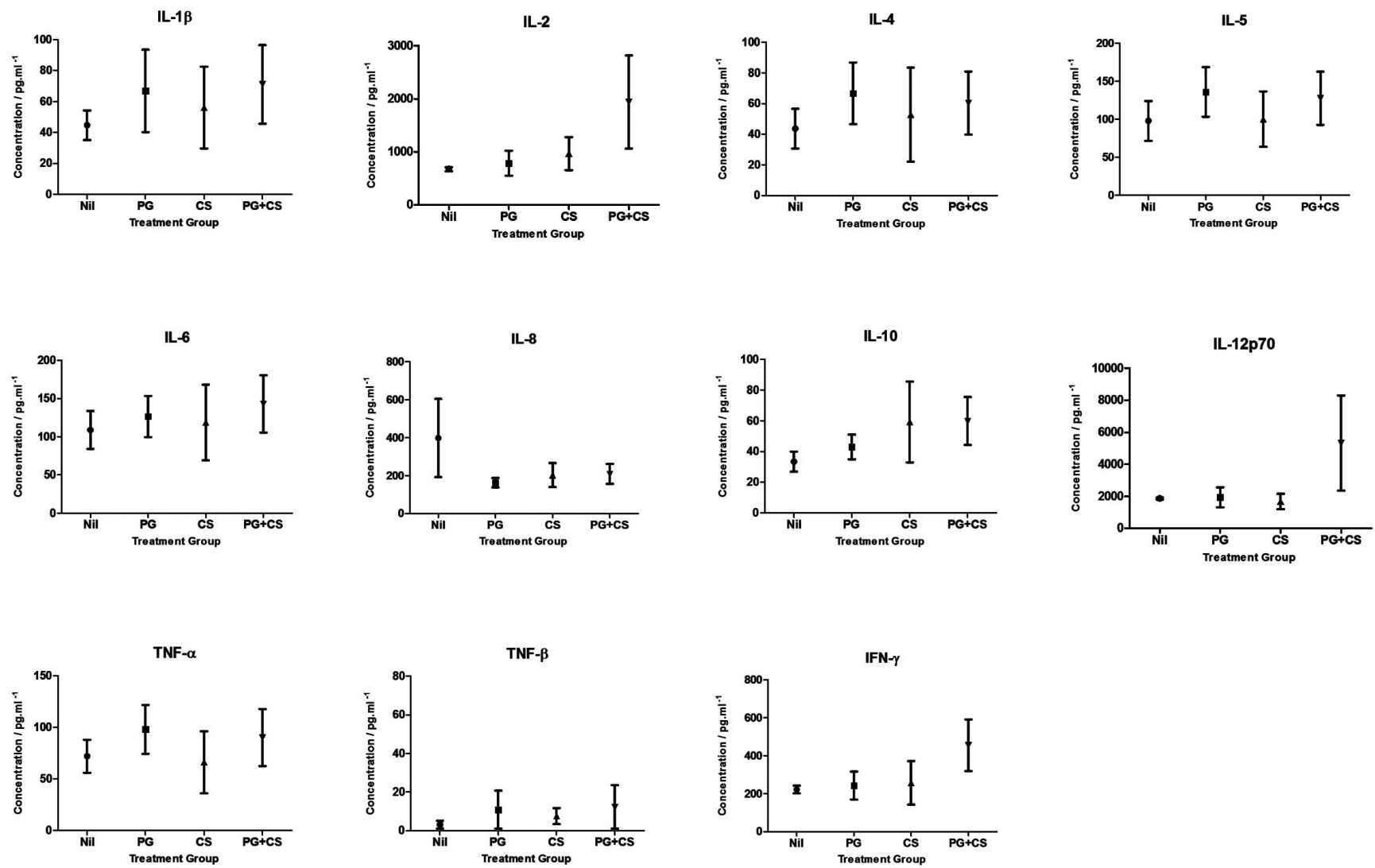

Fig. (2). Tear sample markers of inflammation. Tear samples were obtained using capillary tubes, stained, and assessed using multiplex flow cytometry. Nil, no topical medication; PG, prostaglandin analogues only; CS, corticosteroids only; PG+CS, prostaglandin analogues and corticosteroids concurrently. The mean \pm the standard error of the mean of the detected concentrations is presented. There were no statistically significant differences between the groups.

conjunctival inflammation.

\section{DISCUSSION}

Prostaglandin analogues have always been used with some circumspection in uveitic glaucoma as there have been some reports of exacerbation of uveitis and macular oedema [16], although larger case series have found no evidence of a link [17]. Nevertheless, it is clear that the prostaglandin analogues do cause significant conjunctival hyperaemia [14] and are also potentially proinflammatory [11-13]. They also contain benzalkonium chloride (BAK) as a preservative, which may influence the topical tolerance of these drugs: BAK induces subclinical toxicity and inflammation on the ocular surface and is a major factor responsible for eye drop toxicity in long-term use $[11,18]$.

Conjunctival inflammation is a potential issue in uveitic glaucoma as chronic use of antiglaucoma medication is associated with trabeculectomy failure in primary open-angle glaucoma [6,7], although the reasons for this have not been clearly identified. This study was designed to determine whether prostaglandin analogues are associated with increased levels of conjunctival inflammation in patients with uveitic glaucoma, which would suggest that their use is less desirable in a patient population that is at an increased risk of needing subsequent trabeculectomy surgery.
We found increased expression of inflammatory markers in the impression cytology specimens taken from patients, including HLA-DR, CD54, CCR3 and CCR4, irrespective of the topical medication they were taking. Similar results were seen in the tear film samples that were analysed. Thus it appears that increased expression of conjunctival markers of inflammation and tear film cytokines is primarily driven by the underlying disease rather than by its topical therapy.

Limitations of our pilot study include the small number of patients and the inherent heterogeneity of the clinical presentations of uveitis and raised intraocular pressure. Our study also does not assess the impact of preservatives, particularly BAK $[11,18]$ - as the availability of preservative-free drops increases [19], it may be valuable to evaluate the levels of inflammation in similar patient groups receiving only unpreserved drops in a further study. Nevertheless, we conclude from our pilot study that the topical administration of prostaglandin analogues in patients with uveitic glaucoma is unlikely to increase the level of conjunctival inflammation and is therefore unlikely to prejudice later filtration surgery.

\section{ACKNOWLEDGEMENTS}

The authors gratefully acknowledge the assistance of Grazyna Galatowicz and Virginia Calder PhD of the UCL Institute of Ophthalmology. 


\section{FINANCIAL SUPPORT}

SRJT was supported by the UK National Institute of Health Research and the Imperial College NIHR Comprehensive Biomedical Research Centre. This work was supported by an unrestricted educational grant from Allergan Inc., Irvine, CA, but Allergan had no role in the design or conduct of this research. The authors report no conflicts of interest.

\section{CONFLICT OF INTEREST}

The authors confirm that this article content has no conflicts of interest.

\section{REFERENCES}

[1] Takahashi T, Ohtani S, Miyata K, et al. A clinical evaluation of uveitis-associated secondary glaucoma. Jpn J Ophthalmol 2002; 46(5): 556-62.

[2] Md Din S, Isa H, Taylor SR, et al. Raised intraocular pressure in uveitis. Expert Rev Ophthalmol 2012; 1: 45-59.

[3] Taylor SR, Isa H, Joshi L, et al. New developments in corticosteroid therapy for uveitis. Ophthalmologica 2010; 224 (Suppl 1): 46-53.

[4] de Smet MD, Taylor SR, Bodaghi B, et al. Understanding uveitis: the impact of research on visual outcomes. Prog Retin Eye Res 2011; 30(6): 452-70.

[5] Sallam A, Sheth HG, Habot-Wilner Z, et al. Outcome of raised intraocular pressure in uveitic eyes with and without a corticosteroid-induced hypertensive response. Am J Ophthalmol 2009; 148(2): 207-13 e1.

[6] Broadway DC, Chang LP. Trabeculectomy, risk factors for failure and the preoperative state of the conjunctiva. J Glaucoma 2001; 10(3): 237-49.

[7] Broadway DC, Grierson I, O'Brien C, et al. Adverse effects of topical antiglaucoma medication. II. The outcome of filtration surgery. Arch Ophthalmol 1994; 112(11): 1446-54.

[8] Camras CB. Comparison of latanoprost and timolol in patients with ocular hypertension and glaucoma: a six-month masked, multicenter trial in the United States: The United States Latanoprost Study Group. Ophthalmology 1996; 103(1): 138-47.
[9] Chew PT, Aung T, Aquino MV, et al. Intraocular pressurereducing effects and safety of latanoprost versus timolol in patients with chronic angle-closure glaucoma. Ophthalmology 2004; 111(3): 427-34.

[10] Toris CB, Gabelt BT, Kaufman PL. Update on the mechanism of action of topical prostaglandins for intraocular pressure reduction. Surv Ophthalmol 2008; 53(Suppl1): S107-20.

[11] Pisella PJ, Debbasch C, Hamard P, et al. Conjunctival proinflammatory and proapoptotic effects of latanoprost and preserved and unpreserved timolol: an ex vivo and in vitro study. Invest Ophthalmol Vis Sci 2004; 45(5): 1360-8.

[12] Broadway DC, Grierson I, O'Brien C, et al. Adverse effects of topical antiglaucoma medication. I. The conjunctival cell profile. Arch Ophthalmol 1994; 112(11): 1437-5.

[13] Sharif NA, Kelly CR, Crider JY, et al. Ocular hypotensive FP prostaglandin (PG) analogs: $\mathrm{PG}$ receptor subtype binding affinities and selectivities, and agonist potencies at FP and other PG receptors in cultured cells. J Ocul Pharmacol Ther 2003; 19(6): 501-15.

[14] Alm A, Grierson I, Shields MB. Side effects associated with prostaglandin analog therapy. Surv Ophthalmol 2008; 53(Suppl1): S93-105.

[15] Baudouin C, Bourcier T, Brignole F, et al. Correlation between tear IgE levels and HLA-DR expression by conjunctival cells in allergic and nonallergic chronic conjunctivitis. Graefes Arch Clin Exp Ophthalmol 2000; 238(11): 900-4

[16] Sacca S, Pascotto A, Siniscalchi C, et al. Ocular complications of latanoprost in uveitic glaucoma: three case reports. J Ocul Pharmacol Ther 2001; 17(2): 107-13.

[17] Chang JH, McCluskey $\mathrm{P}$, Missotten $\mathrm{T}$, et al. Use of ocular hypotensive prostaglandin analogues in patients with uveitis: does their use increase anterior uveitis and cystoid macular oedema? $\mathrm{Br}$ J Ophthalmol 2008; 92(7): 916-21.

[18] De Saint Jean M, Brignole F, Bringuier AF, et al. Effects of benzalkonium chloride on growth and survival of Chang conjunctival cells. Invest Ophthalmol Vis Sci 1999; 40(3): 619-30.

[19] Uusitalo H, Pillunat LE, Ropo A. Efficacy and safety of tafluprost $0.0015 \%$ versus latanoprost $0.005 \%$ eye drops in open-angle glaucoma and ocular hypertension: 24-month results of a randomized, double-masked phase III study. Acta Ophthalmol 2010; 88(1): 12-9.

(C) Taylor et al.; Licensee Bentham Open.

This is an open access article licensed under the terms of the Creative Commons Attribution Non-Commercial License (http://creativecommons.org/licenses/by$\mathrm{nc} / 3.0 /$ ) which permits unrestricted, non-commercial use, distribution and reproduction in any medium, provided the work is properly cited. 